\title{
Versatile multipass cell for laser spectroscopic trace gas analysis
}

\section{Journal Article}

\section{Author(s):}

Manninen, A.; Tuzson, B.; Looser, H.; Bonetti, Y.; Emmenegger, L.

Publication date:

2012-11

Permanent link:

https://doi.org/10.3929/ethz-b-000059922

Rights / license:

In Copyright - Non-Commercial Use Permitted

Originally published in:

Applied Physics B 109(3), https://doi.org/10.1007/s00340-012-4964-2 


\title{
Versatile multipass cell for laser spectroscopic trace gas analysis
}

\author{
A. Manninen · B. Tuzson · H. Looser · Y. Bonetti • \\ L. Emmenegger
}

Received: 9 September 2011 / Revised version: 21 November 2011 / Published online: 30 March 2012

(C) Springer-Verlag 2012

\begin{abstract}
The design, construction and characterization of a novel circular multipass cell for sensitive trace gas analysis are presented. This cell allows for easy modification of the optical path length without any changes of its physical parameters. Furthermore, it is suited for three different detection techniques: direct absorption, wavelength modulation and photoacoustics. To demonstrate its performance, mixing ratios of ${ }^{13} \mathrm{CO}_{2}$ and $\mathrm{N}_{2} \mathrm{O}$ were measured from ambient air, using a quantum cascade laser. With the direct absorption method, noise equivalent 1-s precisions of $2.7 \mathrm{ppb}$ and $0.2 \mathrm{ppb}$ are achieved for ${ }^{13} \mathrm{CO}_{2}$ and $\mathrm{N}_{2} \mathrm{O}$, respectively. The wavelength modulation technique resulted in $4.3 \mathrm{ppb}$ precision with 1-s averaging for the ${ }^{13} \mathrm{CO}_{2}$ measurements. A Q-factor of 190 and a normalized noise equivalent minimum absorption of $1.3 \times 10^{-8} \mathrm{~cm}^{-1} \mathrm{~W} \mathrm{~Hz}^{-1 / 2}$ are achieved using the photoacoustic technique.
\end{abstract}

\footnotetext{
A. Manninen $(\varangle) \cdot$ B. Tuzson $\cdot$ L. Emmenegger Laboratory for Air Pollution \& Environmental Technology, Empa, Swiss Federal Laboratories for Materials Science and Technology, Überlandstrasse 129, 8600 Dübendorf, Switzerland e-mail: albert.manninen@empa.ch

Fax: +41-44-8216244

H. Looser

Institute for Aerosol and Sensor Technology, FHNW, University of Applied Sciences, Steinackerstrasse 5, 5210 Windisch, Switzerland

Y. Bonetti

Department of Physics, Institute for Quantum Electronics, ETH

Zürich, Swiss Federal Institute of Technology,

Wolfgang-Pauli-Strasse 16, 8093 Zürich, Switzerland
}

\section{Introduction}

Mid-infrared (IR) laser spectroscopy is widely used to monitor trace gas species in industrial, medical and environmental applications. Mid IR, also known as the spectral fingerprint region, is attractive because the strong fundamental rovibrational bands for most relevant gas molecules allow for high instrumental sensitivity. In this context, quantum cascade lasers (QCLs) are often used as continuous-wave light sources, capable of producing tunable output at room temperature [1].

The selection of the detection method is decisive in the design of any instrument for a specific application. The three most frequently employed spectroscopic techniques in the mid IR are direct absorption (DA), wavelength modulation (WM) as a subgroup of DA and photoacoustics (PA) [2]. The DA technique is rather straightforward, but the signal to noise ratio (SNR) is often limited by laser intensity variations because a small absorption signal is detected on top of a strong background of the laser output. To minimize this background effect, WM can be applied. Most often, the signal is detected at the second-harmonic frequency of the modulation to filter out the background signal at the fundamental frequency. The drawback of WM is its need for more sophisticated electronics to drive the laser and detect the signal. Furthermore, signal analysis to extract the sample concentration from the measured data is more demanding [3]. In the PA method, the absorbed energy is transferred into an acoustic signal and detected by a microphone. Therefore, the detection hardware is rather simple, compared to the optical methods described above. The acoustic signal is only generated in the presence of absorbers; therefore, PA may be considered as an inherently background-free method. But, as the PA signal is proportional to optical power, available lasers are often limiting the sensitivity of the technique. 
The optical path length through a sample gas is crucial to obtain high sensitivity and precision in laser spectroscopy. This has led to a variety of multipass cell configurations, such as White [4], Herriott [5], astigmatic mirror based [6] and variations of cylindrical and spherical mirrors [7-9]. These cells have very stringent requirements for the distance, axial angle and tilt of the mirrors. Although they offer long absorption path lengths, the closing condition (specific circulation pattern of light) is generally accomplished for a selected and fixed parameter set only. Modifying the path length to make the absorption cell suitable for various gas species with different absorption depths usually requires solid knowledge of optics and an experienced person for the alignment and adjustments.

In this work, a versatile multipass cell suitable for the DA, WM and PA techniques is designed. The goals of the design are simplicity of alignment, small detection volume and high sensitivity. The high sensitivity is achieved with a two-dimensional multipass beam pattern, which also acts as a planar excitation for a longitudinal mode of the drum-like PA resonator. The planar light distribution in the cell leads to a small detection volume with robust and easy optical alignment. To complement the unique cell design, a fast data acquisition and processing allowing for $200-\mathrm{Hz}$ real-time data rates was developed.

\section{Instrumentation}

In this section, a short description of the multipass cell design is presented. The first consideration is the size of the sample cell. Minimizing the cell volume is important for building compact instruments and to assure fast gas exchange. However, there is usually a trade-off between minimizing the cell volume and maximizing the absorption signal.

Our concept is based on using six spherical, gold-coated mirrors in a circular configuration. The geometry of the cell is shown in Fig. 1. The six spherical mirrors, each an 8-mmhigh and about 48-mm-wide rectangular slice of a sphere, having $50 \mathrm{~mm}$ radius of curvature, form a nearly continuous ring with approximately $100-\mathrm{mm}$ inner diameter. A $12.6-$ $\mathrm{mm}$ space between two of the mirrors is reserved for coupling optics. In this design, the laser beam bounces back and forth between the mirrors and stepwise advances along the circumference of the cell until it reaches again the coupling lens. Consequently, the maximum number of passes is determined by the beam size and separation of the adjacent reflections. To minimize interference fringes, neighboring reflection spots must not overlap and mirror edges must be avoided.

The circulation pattern is set by the initial aiming onto the cell. A geometrical formulation has been used in Matlab
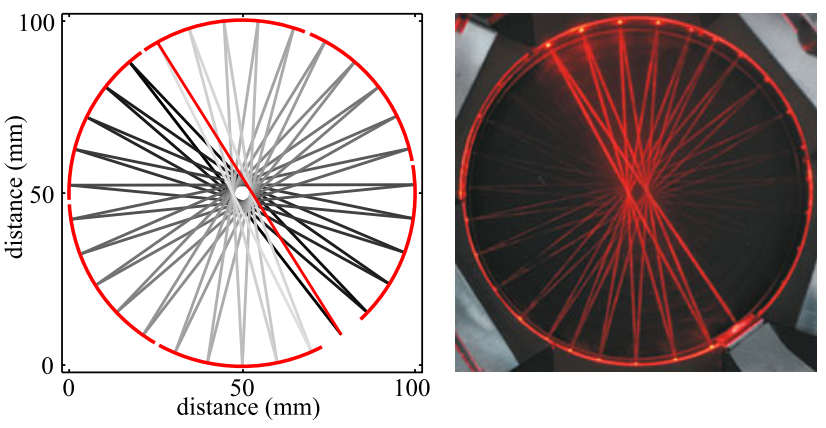

Fig. 1 Calculated (left) and actual (right) beam patterns. An optical path of $310 \mathrm{~cm}$ (31 passes) is achieved with a $2.9^{\circ}$ incidence angle. A $650-n m$ laser is used to visualize the optical path and to align the spherical mirrors

to compute the locations of the beam spots on the mirrors for a variety of input angles. This was done by representing the light as a line and the mirrors as circles, whose centers can be radially shifted in the directions corresponding to the central position of each mirror segment. The reflection points and directions were calculated and optimized for any selected mirror configuration. The beam circulates with a fixed angular advance per pass, producing a star-like structure within the horizontal plane of the cell. Simple rotation of the cell around the vertical axis of the input hole changes the aiming condition, which will induce a wide range of patterns and, thus, path lengths ranging from $0.3 \mathrm{~m}$ (three passes, $\left.30.0^{\circ}\right)$ to $3.7 \mathrm{~m}$ (37 passes, $2.4^{\circ}$ ) can easily be obtained within the $10-\mathrm{cm}$-diameter cell. Any further increase in the number of reflections would require a larger cell diameter to avoid the reflection spots hitting the mirror edges. However, an optical path of $3.7 \mathrm{~m}$ is enough to produce $1 \%$ or more absorption from atmospheric methane, nitrous oxide and the three main isotopologues of carbon dioxide [10].

The beam pattern selected for this study has 31 passes at a $2.9^{\circ}$ input angle, as shown in Fig. 1. The same number of passes can be achieved by changing the input angle to 8.7, 14.5 and even higher values, but increasing the angle of reflection from spherical mirrors will increase the degree of the astigmatic aberration. Astigmatism can be corrected for by shaping the input beam with a cylindrical mirror before coupling into the cell. However, preshaping the input beam compensates for the aberration only at one incidence angle. Thus, in order to sustain flexibility of optical path selection, the pattern produced with the smallest incidence angle without any correction for the astigmatism is preferred in this work.

From the PA point of view, the star pattern also acts as a planar excitation for an acoustic wave. Placing the set of six mirrors in a drum-like acoustic resonator and modulating the laser wavelength across the absorption line at half the frequency of any even-ordered longitudinal eigenmode accumulates acoustic energy and amplifies the signal by a quality factor of the mode [11]. A half-inch microphone (40 HL, 


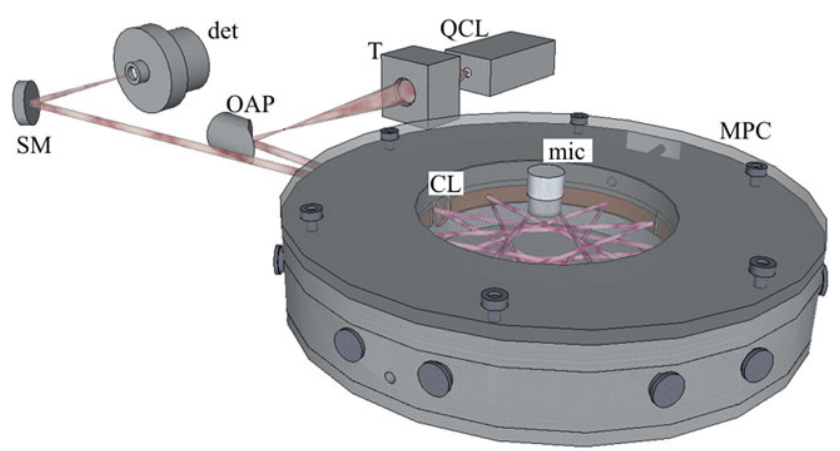

Fig. 2 Instrumentation scheme. Telescope (T), off-axis parabolic mirror (OAP) and coupling lens (CL) are used to shape the QCL beam. The multipass cell (MPC) consists of six spherical mirrors and acts as an acoustic resonator for the PA. A microphone (mic) can be attached to the top of the cell. The output beam is focalized by a spherical mirror (SM) to a detector (det)

G.R.A.S. Sound \& Vibration A/S, Denmark) was fixed to the center of the top cover of the cell to detect the PA signal. It should be noted that the height determined by matching the frequency of the second longitudinal resonance and the peak response of the microphone is $30 \mathrm{~mm}$, which corresponds to $11.6 \mathrm{kHz}$ acoustic frequency, and results in a cell volume of $236 \mathrm{~cm}^{3}$. In contrast, the minimum height of the cell with the DA or WM detection schemes could be as low as $3 \mathrm{~mm}$, resulting in a total volume of $24 \mathrm{~cm}^{3}$.

The QCL (Alpes Lasers SA, Switzerland) used in this study is tunable between $2234 \mathrm{~cm}^{-1}$ and $2244 \mathrm{~cm}^{-1}$ by changing temperature between $0{ }^{\circ} \mathrm{C}$ and $-30{ }^{\circ} \mathrm{C}$. The specified maximum single-mode output power of the laser is $5.6 \mathrm{~mW}$, whereas the optical power reaching the cell is about $2 \mathrm{~mW}$, given the employed laser current and losses in the optical path. The highly divergent laser beam is shaped with a two-mirror beam expander (50105-01, Newport Corp., USA) and a gold-coated off-axis parabolic mirror, as shown in Fig. 2. A plano-convex $\mathrm{CaF}_{2}$ lens, placed between the spherical mirrors, focalizes the beam close to the center of the cell. The custom-made spherical mirrors refocus the beam after each reflection, thus maintaining its size and shape. After multiple reflections from the spherical mirrors, the beam is collimated by the same $\mathrm{CaF}_{2}$ lens. The output light is focalized onto a thermoelectrically cooled detector (PDI-2TE-4.4, Vigo System S.A., Poland).

One purpose of the presented instrumentation was to compare different detection techniques. A LabView program was developed for driving the hardware and processing the detected signals with a special focus to facilitate various laser modulation schemes. For example, in the DA detection, the laser was driven with a current ramp at 2-kHz repetition rate, thus sweeping 2000 times per second over the absorption feature of the gas sample. The laser current was set below the lasing threshold to measure the zero signal after each ramp. Typically, 20 measured scans were averaged, resulting in a $100-\mathrm{Hz}$ spectral data acquisition. The spectra were corrected for the nonlinear frequency tuning of the laser using a prerecorded etalon spectrum, and a fourthorder polynomial baseline was subtracted. The Voigt profile, generated based on spectroscopic parameters from the Hitran database [10] and measured temperature and pressure values, was fitted to the spectra in real time. The fit parameters, i.e. amplitude, width and peak position, were saved for further analysis.

For the WM method, a 100-Hz ramp was superposed by a $100-\mathrm{kHz}$ sinusoidal modulation. A modulation index of 2.2 was used to optimize the SNR in the WM technique, despite the fact that the measured waveform becomes distorted compared to smaller values of the index [12]. The detector signal was measured at the second harmonic of the modulation frequency, i.e. at $200 \mathrm{kHz}$, using a lock-in amplifier (7270, Signal Recovery, USA). The resulting 100-Hz output spectrum was processed in real time similarly to DA, using the second derivative of the Voigt profile, including powermodulation distortion [3].

In the PA technique, typically four modulation waveforms are considered, namely sinusoidal, triangle, quasisquare wave and custom-shaped [13]. The quasi-square wave is considered as the most efficient one, but the wavelength chirp of the laser may degrade the signal at the higher modulation frequencies, which is the case in this work. The custom-shaped modulation requires stable sample conditions and composition, as the modulation waveform is calculated knowing the exact shape of the measured line. Therefore, the triangle modulation, which is more efficient than the sinusoidal one, was selected with an optimal modulation index of 3 . The laser was driven at $5.8 \mathrm{kHz}$ frequency, sweeping the spectral line twice per period. Thus, the PA signal was generated and detected at twice the modulation frequency. Therefore, the acoustic background signal, which originates from the light absorption by the cell walls and thus occurs at the modulation frequency, was separated from the absorption signal of the sample gas in the frequency domain. The microphone output was fed into the lock-in amplifier, averaged over $1 \mathrm{~s}$ and stored. To identify the exact frequency and the profile of the second longitudinal acoustic eigenmode, the PA excitation frequency was swept over the resonance. As a result, the quality factor of the acoustic resonance, defined by the ratio between the central frequency and the FWHM of the power spectrum [14], was determined to be 190. This value is rather high for the longitudinal resonance, because the acoustic damping effect of the cell walls is small for a short but wide resonator.

For all the tested techniques, the cell temperature was stabilized to $30{ }^{\circ} \mathrm{C}$ by means of a heating element, isolation and a temperature controller (WTC3293-14002, Wavelength Electronics Inc., USA). The optical setup was purged with dried and $\mathrm{CO}_{2}$-free air during the measurements. The sample flow through the cell was $0.15 \mathrm{slpm}$, and the pressure 
was kept at $7.2 \mathrm{kPa}$ for the DA and WM measurements, and at $96 \mathrm{kPa}$ for PA.

\section{Results}

The performance of the instrumentation was assessed based on the analysis of spectral lines of $\mathrm{CO}_{2}$ and $\mathrm{N}_{2} \mathrm{O}$ near $2240 \mathrm{~cm}^{-1}$ (see Table 1). The strong ${ }^{13} \mathrm{CO}_{2}$ line (1) was used to characterize the DA, WM and PA techniques, whereas the simultaneous measurements of neighboring lines (2) and (3) were used for long-term ambient air measurements with automated calibration.

The Allan variance is a frequently used statistical tool for characterizing the time-dependent behavior of an experimental instrumentation [15]. Here we adopt the Allan deviation plot where the precision at different averaging times is the ratio between the standard deviation and the mean value of the data. The time series of the DA, WM and PA measurements are shown together with the calculated Allan deviation plots in Fig. 3.

In the case of the DA measurements (Fig. 3, left), the 1-s precision corresponds to $5.2 \times 10^{-8} \mathrm{~cm}^{-1}$ optical path length-normalized noise equivalent $(1 \sigma)$ absorption. The $1 / f$ noise averages down to around $250 \mathrm{~s}$, after which a presumably thermal drift related misalignment of optics and thermal effects of electronics start to dominate the signal. The best precision of the instrumentation presented in this work is comparable with the state-of-the-art results for $\mathrm{CO}_{2}$

Table 1 Spectroscopic characteristics of measured lines at $30^{\circ}$

\begin{tabular}{llll}
\hline Line & Substance & $\begin{array}{l}\text { Line position } \\
\left(\mathrm{cm}^{-1}\right)\end{array}$ & $\begin{array}{l}\text { Line strength } \\
(\text { molecule }\end{array}$ \\
\hline (1) & ${ }^{13} \mathrm{CO}_{2}$ & 2239.399 & $2.09 \times 10^{-21}$ \\
$(2)$ & ${ }^{13} \mathrm{CO}_{2}$ & 2238.979 & $4.16 \times 10^{-22}$ \\
$(3)$ & $\mathrm{N}_{2} \mathrm{O}$ & 2239.062 & $9.30 \times 10^{-19}$ \\
\hline
\end{tabular}

(when difference in the measured line strengths is taken into account) reported in the literature [16]. For the WM, the 1-s noise equivalent absorption is $8.5 \times 10^{-8} \mathrm{~cm}^{-1}$. However, the WM signal deviates from the $1 / \mathrm{f}$ behavior after $10 \mathrm{~s}$, probably due to optical fringes, which affects the fitting procedure in the WM more than in the DA technique. For PA, the random detector noise (microphone and preamplifier) is dominant, and it thus takes about $10^{4} \mathrm{~s}$ to deviate from $1 / \mathrm{f}$ behavior and reach the level of the instrumental drift. The noise equivalent absorption with 1-s averaging for the PA technique is $5.6 \times 10^{-6} \mathrm{~cm}^{-1}$. The precisions for the three measurement techniques are gathered for comparison in Table 2 .

To investigate the influence of the fitting speed on the precision, ramping at $1 \mathrm{kHz}$ was applied with the DA detection. As the maximum real-time fitting speed for the instrumentation is $200 \mathrm{~Hz}$, the individual measured spectra were recorded at the ramping frequency for post processing. The 'playback' mode of the fitting program allows us to test and compare the influence of different fitting parameters, such as speed, with the same measured data set. Therefore, the optimal amount of averaging was assessed based on averaging over 1,10,100 and 1000 spectra before fitting the theoretical prediction to it. As a result, the Allan deviation plots above $1 \mathrm{~s}$ were nearly identical for all the above-mentioned cases, indicating that no systematic distortion of the signal, like $50-\mathrm{Hz}$ jittering of the laser frequency, was present between $1 \mathrm{~Hz}$ and $1 \mathrm{kHz}$. Therefore, the fast fitting before averaging is only important for a fast data rate, not to improve the SNR.

The suitability of the instrumentation for long-term measurements was tested by simultaneous measurement of ${ }^{13} \mathrm{CO}_{2}$ (line 2) and $\mathrm{N}_{2} \mathrm{O}$ (line 3) with the DA method. Ambient air was pumped through the cell with a constant 0.15 slpm flow. Calibration points, alternating between pressurized air and its 1:4 mixture with synthetic air, were measured half hourly for $4 \mathrm{~min}$. The pressurized air contained
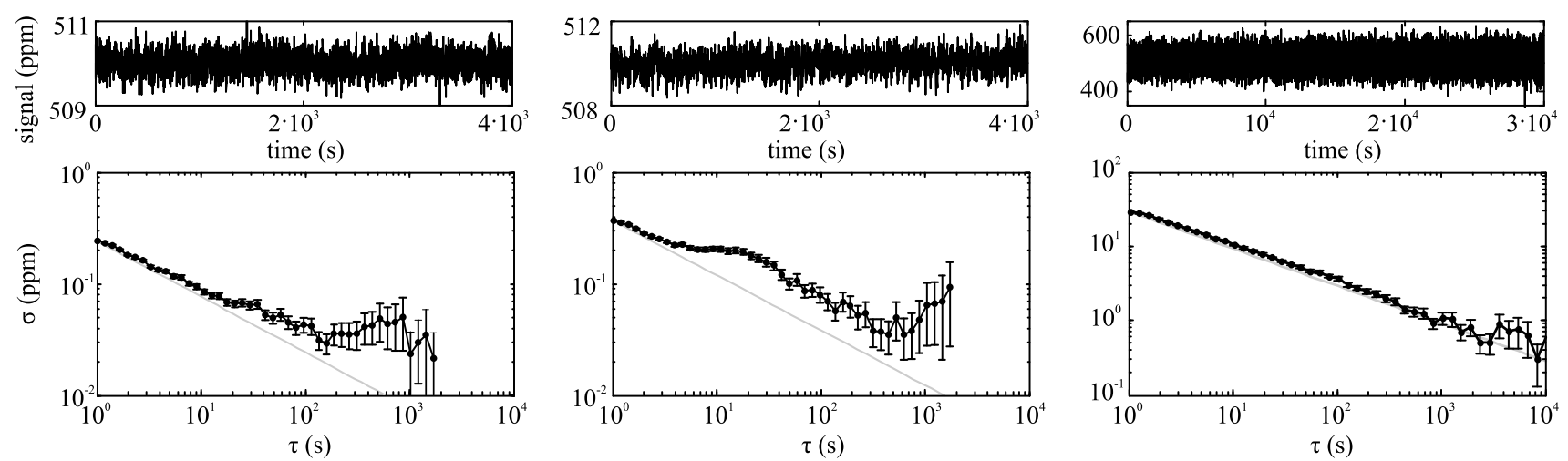

Fig. 3 Measured signals (upper) and calculated Allan deviations (lower) of $\mathrm{CO}_{2}$ with the DA (left), WM (center) and PA (right) techniques. Measurement precisions at 1 -s averaging are $0.47 \%, 0.76 \%$ and $5.79 \%$, respectively. Gray line shows 1/f noise behavior 
Table 2 Precisions (in \%) of $510 \mathrm{ppm} \mathrm{CO}_{2}$ mixing ratio measurements

\begin{tabular}{lcl}
\hline Method & Precision at $1 \mathrm{~s}$ & Best precision (time/s) \\
\hline DA & 0.47 & $0.05(250)$ \\
WM & 0.76 & $0.07(450)$ \\
PA & 57.94 & $0.60(8000)$ \\
\hline
\end{tabular}
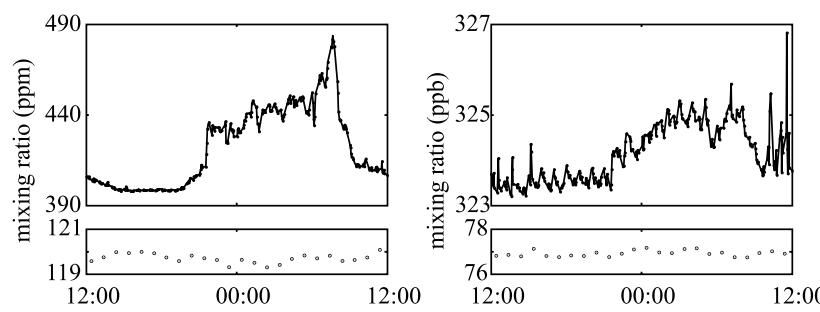

Fig. $4 \mathrm{CO}_{2}$ (left) and $\mathrm{N}_{2} \mathrm{O}$ (right) measurements from outside air. Dots represent 3-min averages. Diluted calibration gas over $24 \mathrm{~h}$ (bottom graphs), having standard deviations of $0.2 \mathrm{ppm}$ and $0.1 \mathrm{ppb}$ for $\mathrm{CO}_{2}$ and $\mathrm{N}_{2} \mathrm{O}$, respectively

$510 \mathrm{ppm}$ and $335 \mathrm{ppb}$ of $\mathrm{CO}_{2}$ and $\mathrm{N}_{2} \mathrm{O}$, respectively. The diluted calibration gas was used as an external standard to test the accuracy of the long-term measurement. A driftcorrection factor was determined using the undiluted calibration gas measurements and applied to the raw data. The determined mixing ratios over $24 \mathrm{~h}$ are shown in Fig. 4, where natural abundance of $1.106 \%$ was assumed for the ${ }^{13} \mathrm{CO}_{2}$ isotopologue.

The diurnal cycle can be easily seen for $\mathrm{CO}_{2}$, while for $\mathrm{N}_{2} \mathrm{O}$ it is much smaller, but still resolvable within the precision of the measurement. It should be recognized that the standard deviations of the diluted calibration gas in the bottom graphs of Fig. 4 also include the contribution of the gas mixing error.

\section{Discussion and outlook}

The spectral tuning of QCLs is mainly controlled by the temperature, which affects the effective refractive index of the semiconductor medium; direct current tuning is negligible, compared to bipolar laser diodes [17]. Therefore, the change of the modulation current is followed by the wavelength change at lower speed, resulting in pronounced wavelength chirp at the beginning of temperature change. To reduce this effect, the initial part of the signal, recorded at the beginning of the ramp, is not used for spectral fitting. The heat dissipation from the laser chip is considerably slower than the current-induced heating of the laser, resulting in tunability hysteresis at higher modulation frequencies. This has only little effect on the DA signal, as the ramp is asymmetric, relatively slow and monotonically increasing. However, it affects significantly the PA and WM techniques by shifting part of the useful signal from the second-harmonic frequency.

The study and comparison of different detection techniques makes it possible to tailor the instrument to fit a wide range of applications. Therefore, future development of the instrument will include selection of the most appropriate detection scheme for any specific application.

For the DA method, the signal detection and processing are relatively simple, although the technique does require rather expensive and wavelength-dependent mid-IR detectors and careful optimization of the light path. However, the low sample pressure and much smaller detection volume than required for the PA detection make it possible to downsize the cell volume to $24 \mathrm{~cm}^{3}$, assuming a cell height of $3 \mathrm{~mm}$. With such a small volume, a very high sample exchange rate of up to $100 \mathrm{~Hz}$ can be achieved.

The precision of the WM measurements is comparable to the DA technique. This method requires relatively complex data treatment, in addition to excellent optical alignment, fast detection and additional electronics. The aforementioned low sample volume with fast exchange rate is also applicable to the WM technique. Furthermore, improving the fitting procedure by including the large modulation index related distortion should further improve the performance of this technique. Applying harmonic normalization or higher modulation frequencies could improve the sensitivity of the method, albeit at the cost of requiring additional hardware being introduced to the instrumentation [18, 19].

The PA technique has some inherent advantages, since it does not depend on optical detection. The alignment of the optical path is not very critical because the light beam does not have to be focused onto a detector element. Also, interference fringes, which are often limiting the detectivity in DA and WM, are of very minor importance in PA, allowing for longer optical path lengths compared to the aforementioned techniques. The design described in this work is especially suited for PA, because the cell can be machined from one solid piece, which eliminates discontinuities in the cell walls and thus improves the acoustic amplification. Although less sensitive than DA or WM in our comparison, the detectivity could be significantly enhanced by future developments in both lasers and microphones. Since the PA signal is directly proportional to the excitation power, already available more powerful QCLs would increase the sensitivity. Furthermore, electromechanical film microphone stacks at both ends of the resonator can be used to further improve acoustic detection due to a larger size microphone and a differential detection $[20,21]$.

The next development steps will be constituted by compacting the instrument's size to significantly smaller dimensions and selecting the appropriate detection technique for specific application. Planned exchange of the laser with one emitting near $2310 \mathrm{~cm}^{-1}$ will improve the performance of 
the presented instrumentation due to stronger lines of $\mathrm{CO}_{2}$. Consequently, this will enable simultaneous measurements of all three main stable $\mathrm{CO}_{2}$ isotopic species, i.e. ${ }^{12} \mathrm{C}-\mathrm{CO}_{2}$, ${ }^{13} \mathrm{C}-\mathrm{CO}_{2}$ and ${ }^{18} \mathrm{O}-\mathrm{CO}_{2}$ [22]. However, the sensitivity of the high-frequency PA technique is expected to be reduced by a near-resonant collisional energy transfer between the excited $\mathrm{CO}_{2}$ and a metastable first vibrational level of $\mathrm{N}_{2}$ at $2330.7 \mathrm{~cm}^{-1}$ [23-25]. On the other hand, $\mathrm{H}_{2} \mathrm{O}$ in the sample gas acts as a catalyst to speed up vibrational relaxation of molecules, thus increasing the PA signal $[26,27]$. Therefore, extra care must be taken in determining the sample matrix in order to reliably extract the $\mathrm{CO}_{2}$ concentration with the PA method near $2310 \mathrm{~cm}^{-1}$.

Further possibilities to take advantage of the presented cell design include the use of multiple lasers [28] which could be aligned such that they produce the same or different optical path lengths within the same sample volume. Also, the Stark-modulation approach [29] could be easily implemented, since the planar ends of the cell may be used as electrodes to generate and modulate a homogeneous electric field.

\section{Conclusions}

A circular optical cell, suitable for multipass direct absorption, wavelength modulation and resonant photoacoustic techniques for sensitive trace gas measurements, is presented. Its performance is demonstrated by the quantitative measurements of $\mathrm{CO}_{2}$ and $\mathrm{N}_{2} \mathrm{O}$ at ambient concentrations. Noise equivalent $1-\mathrm{s}$ precision of $2.7 \mathrm{ppb}$ and minimum absorption of $5.6 \times 10^{-9} \mathrm{~cm}^{-1}$ with 250 -s averaging for ${ }^{13} \mathrm{CO}_{2}$ are achieved with DA, whereas 1-s precision of $4.3 \mathrm{ppb}$ and minimum absorption of $7.8 \times 10^{-9} \mathrm{~cm}^{-1}$ with 450-s averaging are determined for the WM technique. With PA, a normalized noise equivalent absorption (NNEA) of $1.3 \times 10^{-8} \mathrm{~cm}^{-1} \mathrm{~W} \mathrm{~Hz}^{-1 / 2}$ is achieved. Sensitivity, (isotopic) selectivity and real-time spectral fitting of up to $200 \mathrm{~Hz}$ make this instrumentation attractive for many applications, including eddy covariance flux measurements, breath air analysis and industrial process and environmental monitoring. Future development will include more compact and integrated optics, thermal stabilization, customized electronics and improvements in the design for industrial production.

Acknowledgements The authors would like to acknowledge the SNSF, the Swiss Confederation and NanoTera (www.nano-tera.ch) for scientific evaluation and financial support. Alpes Lasers SA is acknowledged for providing the quantum cascade laser.

\section{References}

1. R.F. Curl, F. Capasso, C. Gmachl, A.A. Kosterev, B. McManus, R. Lewicki, M. Pusharsky, G. Wysocki, F.K. Tittel, Chem. Phys. Lett. 487, 1 (2010)

2. W. Demtröder, Laser Spectroscopy, Basic Concepts and Instrumentation (Springer, Berlin, 1981)

3. P. Kluczynski, J. Gustafsson, Å.M. Lindberg, O. Axner, Spectrochim. Acta B 56, 1277 (2001)

4. J.U. White, J. Opt. Soc. Am. 32, 285 (1942)

5. D. Herriott, H. Kogelnik, R. Kompfner, Appl. Opt. 3, 523 (1964)

6. J.B. McManus, P.L. Kebabian, M.S. Zahniser, Appl. Opt. 34, 3336 (1995)

7. S.M. Chernin, E.G. Barskaya, Appl. Opt. 30, 51 (1991)

8. J. Silver, Appl. Opt. 44, 6545 (2005)

9. Y. Kato, J. Sato, T. Nakamura, M. Endo, S. Yamaguchi, K. Nanri, T. Fujioka, in Lasers and Electro-Optics-Pacific Rim, 2007, Seoul, Korea (CLEO, Pacific Rim, 2007), p. 1

10. L.S. Rothman, I.E. Gordon, A. Barbe, D.C. Benner, P.F. Bernath, M. Birk, V. Boudon, L.R. Brown, A. Campargue, J.-P. Champion, K. Chance, L.H. Coudert, V. Dana, V.M. Devi, S. Fally, J.-M. Flaud, R.R. Gamache, A. Goldman, D. Jacquemart, I. Kleiner, N. Lacome, W.J. Lafferty, J.-Y. Mandin, S.T. Massie, S.N. Mikhailenko, C.E. Miller, N. Moazzen-Ahmadi, O.V. Naumenko, A.V. Nikitin, J. Orphal, V.I. Perevalov, A. Perrin, A. Predoi-Cross, C.P. Rinsland, M. Rotger, M. Šimečková, M.A.H. Smith, K. Sung, S.A. Tashkun, J. Tennyson, R.A. Toth, A.C. Vandaele, J.V. Auwera, J. Quant. Spectrosc. Radiat. Transf. 110, 533 (2009)

11. A. Rosencwaig, Photoacoustics and Photoacoustic Spectroscopy (Krieger, Malabar, 1980)

12. J. Reid, D. Labrie, Appl. Phys. B 26, 203 (1981)

13. J. Saarela, J. Toivonen, A. Manninen, T. Sorvajärvi, R. Hernberg, Appl. Opt. 48, 743 (2009)

14. C.F. Dewey, R.D. Kamm, C.E. Hackett, Appl. Phys. Lett. 23, 633 (1973)

15. D.W. Allan, Proc. IEEE 54, 221 (1966)

16. J.B. McManus, D.D. Nelson, M.S. Zahniser, Isot. Environ. Health Stud. 46, 49 (2010)

17. F. Capasso, Opt. Eng. 49, 111102 (2010)

18. J.A. Silver, Appl. Opt. 31, 707 (1992)

19. D.T. Cassidy, J. Reid, Appl. Opt. 21, 1185 (1982)

20. A. Manninen, J. Sand, J. Saarela, T. Sorvajärvi, J. Toivonen, R. Hernberg, Opt. Express 17, 16994 (2009)

21. J. Saarela, T. Sorvajärvi, T. Laurila, J. Toivonen, Opt. Express 19, A725 (2011)

22. B. Tuzson, J. Mohn, M.J. Zeeman, R.A. Werner, W. Eugster, M.S. Zahniser, D.D. Nelson, J.B. McManus, L. Emmenegger, Appl. Phys. B 92, 451 (2008)

23. G. Herzberg, Molecular Spectra and Molecular Structure I. Spectra of Diatomic Molecules (Van Nostrand, New York, 1950)

24. J.E. Morgan, H.I. Schiff, Can. J. Chem. 41, 903 (1963)

25. C.K.N. Patel, Phys. Rev. Lett. 13, 617 (1964)

26. R.L. Taylor, S. Bitterman, Rev. Mod. Phys. 41, 26 (1969)

27. P.L. Meyer, M.W. Sigrist, Rev. Sci. Instrum. 61, 1779 (1990)

28. C.G. Tarsitano, C.R. Webster, Appl. Opt. 46, 6923 (2007)

29. C. Dyroff, A. Zahn, W. Freude, B. Jänker, P. Werle, Appl. Opt. 46, 4000 (2007) 